\title{
Experiments and simulations on the possibility of radiative contraction/collapse in the PF-1000 plasma focus
}

\author{
Mohamad Akel, \\ Jakub Cikhardt, \\ Pavel Kubes, \\ Hans-Joachim Kunze, \\ Sing Lee, \\ Marian Paduch, \\ Sor Heoh Saw
}

\begin{abstract}
Experimental studies of discharges in the plasma focus facility with neon filling and respective numerical simulations employing the radiative Lee code are reported. The pinch currents exceed the Pease-Braginskii current, which indicates that radiative losses are larger than heating and that contraction of the formed plasma should occur. Both of these effects were indeed observed. Parallel numerical simulations were crucial for the identification of such an effect.
\end{abstract}

Key words: plasma focus $\bullet$ radiative collapse $\bullet$ radiation losses $\bullet$ simulations

M. Akel

Department of Physics,

Atomic Energy Commission of Syria,

Damascus, P. O. 6091, Syria

J. Cikhardt, P. Kubes

Czech Technical University in Prague,

Technická 2, 16627 Prague, Czech Republic

H. -J. Kunze

Institute for Experimental Physics V,

Ruhr-University,

Bochum, Germany,

Tel.: +4923432 25785,

E-mail: Hans-Joachim.Kunze@rub.de

S. Lee

INTI International University,

Nilai, Malaysia

M. Paduch

Institute of Plasma Physics and Laser Microfusion,

23 Hery Str., 01-497 Warsaw, Poland

S. H. Saw

Nikai University,

71800 Nilai, Malaysia

Received: 18 September 2015

Accepted: 14 November 2015

\section{Introduction}

The possibility of radiative collapse in pinched linear discharges was first studied independently by Pease [1] and Braginskii [2] in 1957 for pure hydrogen or deuterium plasmas. When radiation losses by bremsstrahlung exceed Ohmic heating at high currents, they predicted shrinking of the plasma column, which may even result in a collapse of the column to extremely high densities. They derived a critical current value that has to be exceeded. Originally calculated for homogeneous and infinitely long plasmas, refined approaches considered later took into account, for example, density profiles, axial outflow of plasma [3], which occurs in all laboratory plasmas of finite length, anomalous resistivity, opacity, and coupling of the pinch formation into a realistic electrical circuit [4].

For the observation of the phenomenon, $z$-pinches were studied on the experimental side, also specifically the pinch plasmas formed from fibers of frozen hydrogen or deuterium and driven by high-voltage pulse generators. No radiative collapse or at least contraction could be clearly identified. This could be due to, for example, the formation of complex internal structures like magnetic flux loops and filaments [5]. Only one group reports the attainment of the Pease-Braginskii current in an ultra-high-pressure discharge [6].

Linear pinch plasmas are also produced in discharges of the plasma focus type. Discharges with 
small amounts of heavy impurity gases lead to the formation of small high-density hot spots, which were interpreted as result of a local radiative collapse caused by strong line radiation losses and outflow of plasma from the neck regions of an $m=0$ instability [7]. Here we report the first investigations to study radiative contraction/collapse in the plasma focus device PF-1000 experimentally combined with numerical simulations.

\section{Collapse model}

Pease and Braginskii started from a homogenous pinch plasma in the Bennett equilibrium, which is independent of the plasma radius $r_{p}$ :

$$
(1+Z) N_{i} k T=\frac{\mu_{0}}{4 \pi} I^{2}
$$

where $N_{i}$ is the number of ions per unit length, $T_{i}=$ $T_{\mathrm{e}}=T$, and $Z$ is the charge of the ions. The plasma radius $r_{p}$ depends on the energy balance between Joule heating $P_{I}$ and radiation losses by bremsstrahlung $P_{\text {rad. }}$. For $P_{\text {rad }}=P_{I}$ and a Spitzer resistivity in the plasma, the specific current now known as a Pease-Braginskii current $I_{\mathrm{PB}}$ becomes

$$
\frac{I_{\mathrm{BP}}}{\mathrm{MA}} \approx 0.27 \sqrt{\ln \Lambda}\left(1+\frac{1}{Z}\right)
$$

A weak temperature dependence is through the Coulomb logarithmus $\ln \Lambda$. With no power balance, the energy equation yields [4]

$$
\frac{1}{r} \frac{\mathrm{d} r}{\mathrm{~d} t}=\frac{3}{4} \frac{1}{r_{\mathrm{rad}}}\left(\frac{I_{\mathrm{BP}}^{2}}{I^{2}}\right)
$$

where $I_{\mathrm{PB}}$ is a critical current and $\tau_{\mathrm{rad}}$ is the time constant for energy loss by radiation,

$$
\tau_{\text {rad }}=\frac{(3 / 2)(1+Z) N_{i} k T}{P_{\text {rad }}}
$$

It is obvious that for a plasma current $I<I_{\mathrm{PB}}$, we have expansion of the plasma column and for $I>I_{\mathrm{PB}}$ shrinking of the same column. In plasmas of heavy gases, emitted line radiation $P_{\text {line }}$ exceeds strongly bremsstrahlung emission and thus reduces not only the critical Pease-Braginskii current but also highly shortens the radiation loss time $\tau_{\text {rad }}$, and hence also the speed of contraction (Eq. (3)).

Of course, this simple model does not realistically describe the formation of the plasma in PF devices, since specifically there is no pressure equilibrium reached and no other effects mentioned above are accounted for. It is revealed through Eq. (3) that the dependence of any effect that matters is on two quantities: $I_{\mathrm{BP}}$ and $\tau_{\text {rad. }}$ Although changed in realistic plasmas, the above Pease-Braginskii current (Eq. (2)) remains a useful indicator for the range where the radiation effects might be observable.

Table 1 gives $I_{\mathrm{BF}}$ for the gases used in the discharges reported below. The second column shows the temperature of the completely ionized gas, the third column the temperature at which $50 \%$ are completely stripped.
Table 1. Gas fillings used in this study and Pease-Baginskii current $I_{\mathrm{BP}}$

\begin{tabular}{lccc}
\hline Species & $\begin{array}{c}\text { Fully ionized } \\
\text { at }\end{array}$ & $\begin{array}{c}50 \% \text { stripped } \\
\text { at }\end{array}$ & $\begin{array}{c}I_{\mathrm{PB}} \\
{[\mathrm{MA}]}\end{array}$ \\
\hline $\mathrm{H} / \mathrm{D}$ & $40 \mathrm{eV}$ & & 1.70 \\
$\mathrm{He}$ & $160 \mathrm{eV}$ & $30 \mathrm{eV}$ & 1.27 \\
$\mathrm{Ne}$ & $4000 \mathrm{eV}$ & $680 \mathrm{eV}$ & 0.94 \\
\hline
\end{tabular}

\section{Simulation of the plasma formation in plasma focus discharges}

The plasma development in plasma focus discharges of the Mather type can be simulated employing the five-phase Lee model code of the Mather type [8].

It couples the electrical circuit with the plasma focus dynamics, thermodynamics, and radiation. Inputs are electric circuit parameters, tube parameters, charging voltage, gas parameters, and filling pressure. Then a calculated current waveform is fitted to an experimental waveform by four model parameters in order to have a realistic simulation. The code gives several parameters, the ones of interest here are the trajectories of shock wave, the piston, and the minimum radius of the finally formed plasma column. For discharges in hydrogen and in inert gases, conditions of radiative cooling and collapse in PF-1000 were studied and are being published [9]. Start of the pinch and definition of pinch radius $r_{p}$ are taken from the time when the piston and reflected shock meet. For other plasma focus devices, similar studies were reported earlier, e.g. in [10].

\section{Experiments}

The experiments were performed at the Mather-type plasma focus facility PF-1000 [11]. The present characteristics and parameters of operation have been published in a number of papers, e.g. in [12]. Recent typical charging voltages were $23 \mathrm{kV}$ driving a maximum current of (1.7-1.9) MA. The pinch current $I_{\text {pinch }}$, which has to be compared with the Pease-Braginskii current in the last column of Table 1, is about two-thirds of $I_{\mathrm{PB}}$, i.e. about $1.2 \mathrm{MA}$. We see, therefore, that contraction or even collapse should not be expected for hydrogen plasmas. Helium plasmas are at the boundary, but for neon plasmas, the pinch current should be sufficiently above $I_{\mathrm{PB}}$. In the actual experiments, this threshold current will be even lower due to the additional losses by line radiation.

The relevant parameter for our studies is the minimum plasma radius $r_{\min }$. It was obtained from multiframe interferometric measurements of the plasma column, employing the second harmonic $(527 \mathrm{~nm})$ of a Nd:YLF laser of less than $1 \mathrm{~ns}$ duration. The laser pulse was split by mirrors into 15 separated beams, which passed through a Mach-Zehnder interferometer [13]. Table 2 shows some results. The first column gives the discharge number and the next columns the discharge parameters. Column $d$ displays the minimum diameter $2 r_{\min }$ of 
Table 2. Results from experiments and simulations

\begin{tabular}{lccccccccc}
\hline \multicolumn{1}{c}{ Shots } & $\begin{array}{c}\text { No. of } \\
\text { shots }\end{array}$ & Gas & $\begin{array}{c}p \\
{[\text { Torr }]}\end{array}$ & $\begin{array}{c}U \\
{[\mathrm{kV}]}\end{array}$ & $\begin{array}{c}I \\
{[\mathrm{MA}]}\end{array}$ & $\begin{array}{c}I_{\text {pinch }} \\
{[\mathrm{MA}]}\end{array}$ & $\begin{array}{c}D \\
{[\mathrm{~cm}]}\end{array}$ & $r_{\min } / a$ & $\begin{array}{c}k=r_{\min } / a \\
\text { simul. }\end{array}$ \\
\hline $10095 \mathrm{ff}$ & $3 \mathrm{av}$ & $\mathrm{Ne}$ & 0.5 & 23 & 1.7 & 1.14 & 0.53 & 0.023 & 0.13 \\
$10125 \mathrm{ff}$ & 4 & $\mathrm{Ne}+\mathrm{D}_{2}$ & 0.55 & 23 & 1.7 & 1.20 & 0.53 & 0.023 & 0.12 \\
$10103 \mathrm{ff}$ & 3 av. & $\mathrm{Ne}+\mathrm{D}_{2}$ & 0.8 & 23 & 1.8 & 1.33 & 0.57 & 0.024 & 0.09 \\
10112 & 1 & $\mathrm{Ne}+\mathrm{D}_{2}$ & 1.0 & 23 & 1.95 & 1.38 & 0.75 & 0.032 & 0.05 \\
$10063 \mathrm{ff}$ & 4 & $\mathrm{D}_{2}$ & 1.5 & 23 & 1.8 & 1.25 & 1.40 & 0.061 & \\
10630 & 1 & $\mathrm{D}_{2}$ & 1.5 & 21 & 1.7 & 1.20 & 1.30 & 0.057 & 0.19 \\
10915 & 2 & $\mathrm{He}$ & 0.9 & 16 & 1.2 & 0.90 & 1.00 & 0.044 & 0.18 \\
\hline
\end{tabular}

the pinched plasma and column $k=r_{\min } / a$ the radius normalized to the radius a of the anode.

Unfortunately, in two experimental attempts in the past year, the PF-1000 facility could not be operated at sufficiently high charging voltages, and only a few usable results were obtained for D and He. For the analysis of neon discharges, we, therefore, utilized earlier experiments also in the above table, which had been already analyzed under different aspects and are reported in parts there [14].

The last column of Table 2 gives the normalized minimum radius $k$ from the simulations for the respective discharge. For the $\mathrm{D}_{2}$ and He discharges, the experimental values of $k$ are about three to four times lower than the simulation result. One possible reason for that could be the strong plasma outflow along the axis caused by the zippering effect, which is clearly seen in the interferograms. The experimental radii of Table 2 were therefore taken close to anode.

Figure 1 shows the value of $k$ from the simulations of the neon discharges as a function of filling pressure together with the experimental observations. The experimental values (a) are lower than the results from the simulations (b). Although one could again invoke plasma losses due to the zippering effect or beams, the large difference of 0.5 Torr is surprising. The simulations predict the strongest influence of radiation losses for discharges around 1 Torr filling pressure whereas the experiments indicate this to be for half the pressure. Future measurements at lower and higher pressures should resolve this discrepancy.

To identify the influence of the radiation losses, simulations were also carried out without them, curve (c): the influence is clearly seen in the minimum pinch radius. The combination of experiment and simulation, where terms can be used or easily

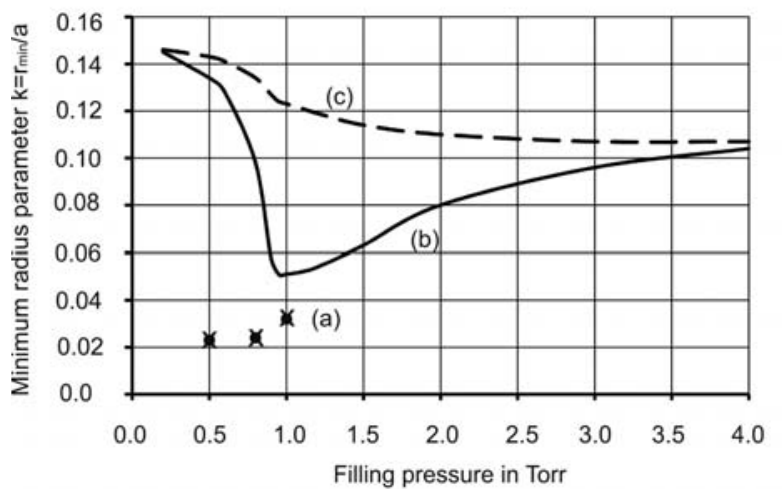

Fig. 1. Normalized minimum plasma radius $k$ for Ne discharges: (a) experimental values, (b) simulation with radiative losses, and (c) simulation without radiative losses. discarded, is thus a unique tool in the analysis of these studies.

\section{Conclusion}

Investigations of the minimum plasma radius in the plasma focus facility PF-1000 operated with neon filling point to the influence of line radiation on the contraction of the pinched plasma column. Simulations by the radiative Lee code, which specifically models the plasma dynamics till the final pinched plasma and also further in discharges of the plasma focus type, support the interpretation. For the studied discharges with neon no collapse and only shrinking occurred.

Acknowledgments. MA thanks the Director General of AECS, for encouragement and permanent support. CJ, PK and MP acknowledge support by the Research Programme under MSMT nos. LG15013, LH13283, IAEA RC-16954, RC-16956, RC-17088, RC-17165, SGS 13/194/OHK3/3T/13 and by the Polish Ministry of Science and Higher Education within the framework of the financial resources in 2015 allocated for the realization of the international cofinanced projects. SL and SHS thank for support by the research grants INT-CPR-01-02-2012 and FRGS/2/2013/SG02/INTI/01/1.

This work was performed at the AEC, Syria, CTU, Czech Republic, RUB, Germany, INTI International University, Malaysia, and IPPLM, Poland.

\section{References}

1. Pease, R. S. (1957). Equilibrium characteristics of a pinched gas discharge cooled by bremsstrahlung radiation. Proc. $R$. Soc. London Ser. B, 70, 11-13.

2. Braginskii, S. I. (1957). Contribution to the theory of spark channels. Sov. Phys. JETP, 6, 494-501.

3. Bernal, I., \& Bruzonne, H. (2002). Radiative collapses in Z-pinches with axial mass losses. Plasma Phys. Control. Fusion, 44, 223-231.

4. Robson, A. E. (1989). Radiative collapse of a Z pinch in hydrogen and helium. Phys. Fluids $B, 1(1)$, 1834-1842.

5. Rudakov, L. I., \& Sudan, R. N. (1997). MHD turbulence in radiating Z-pinches. Phys. Rep., 283, 53-63.

6. Bogomaz, A. A., Budin, A. V., Losev, S. Yu., Pinchuk, M. E., Pozubenkov, A. A., Rutberg, Ph. G., \& Savvatev, A. F. (2008). Attainment of the Pease-Braginskii current in an ultra-high-pressure discharge. Plasma Phys. Rep., 34, 366-375. 
7. Koshelev, K. N., Krauz, V. I., Reshetniak, N. G., Salukvadze, R. G., Sidelnikov, Yu. V., \& Khautiev, E. Yu. (1988). The formation of the micropinch structure in plasma focus by the addition of heavy impurities. $J$. Phys. D-Appl. Phys., 21, 1827-1829.

8. Lee, S. (2014). Plasma Focus radiative model: Review of the Lee model code. J. Fusion Energy, 33, 319-335.

9. Lee, S., Saw, S. H., Akel, M., Kunze, H. -J., Kubes, P., \& Paduch, M. (2016). Conditions for radiative cooling and collapse in the Plasma Focus illustrated with numerical experiments on the PF-1000. IEEE Trans. Plasma Sci., 44, 165-173.

10. Akel, M., \& Lee, S. (2013). Radiative collapse in Plasma Focus operated with heavy noble gases. $J$. Fusion Energy, 32, 111-116.

11. Scholz, M., Miklaszewski, R., Gribkov, V. A., \& Mezzetti, F. (2000). PF-1000 device. Nukleonika, 45(3), 155-158.
12. Kubes, P., Paduch, M., Cikhardt, J., Kravarik, J., Rezac, K., Kortanek, J., Cikhardtova, B., \& Zielinska, E. (2015). Existence of a return direction for plasma escaping from a pinched column in a plasma focus discharge. Phys. Plasmas, 222, 052706.

13. Zielinska, E., Paduch, M., \& Scholz, M. (2011). Sixteen-frame interferometer for a study of a pinch dynamics in PF-1000 device. Contrib. Plasma Phys., 51, 279-283.

14. Kubes, P., Paduch, M., Cikhardt, J., Kortanek, J., Cikhardtova, B., Rezac, K., Klir, D., Kravarik, J., \& Zielinska, E. (2014). Filamentary structure of plasma produced by compression of puffing deuterium by deuterium or neon plasma sheath on plasma-focus discharge. Phys. Plasmas, 21, 122706. 